\title{
Structure and Properties of Tapered Block Polymers of Styrene and Isoprene II. Dynamic Mechanical Responses and Their Structural Interpretations
}

\author{
Takeji Hashimoto, ${ }^{*}$ Yasuhisa TsuKahara, ${ }^{a}$ and Hiromichi KawaI ${ }^{\mathrm{b}}$ \\ Department of Polymer Chemistry, Faculty of Engineering, \\ Kyoto University, Kyoto 606, Japan
}

(Received April 1, 1983)

\begin{abstract}
The dynamic mechanical properties of "tapered" block polymers consisting of styrene and isoprene were studied in relation to the unique microdomain structure of these polymers. It was found from morphological observations that considerable mixing of the two monomers occurs in the primary structure, giving rise to a class of block polymers consisting approximately of "styrene-rich" and "isoprene-rich" block chains. Microphase-separated domain structures existed in the tapered block polymers prepared by sequential living anionic polymerization. Each domain, however, contained a substantial amount of dissimilar monomeric units, thus having an order-to-disorder transition temperature $T_{\mathrm{c}}$ (the temperature at which the microdomains are dissolved into a homogeneous mixture) lower than that of the ideal block. This lowering in $T_{\mathrm{c}}$ should have a substantial influence on flow behavior. The tapered block polymers gave essentially a single broad primary dispersion. This dispersion is related to the microbrownian motion of segments with a relaxation time between those of the parent homopolymers. The temperature dependence of the shift factor $a_{\mathrm{T}}$ obeyed the WLF equation, in spite of the heterophase structure. The mechanical behavior was interpreted using a model in which two incompatible units undergo extensive mixing in each domain, leading to an increase in the interfacial volume fraction.

KEY WORDS Block Polymer / Tapered Block Polymer / Microdomain Structure / Dynamic Mechanical Properties / WLF Equation / Microphase Separation / Order-Disorder Transition / Critical Temperature / SmallAngle X-Ray Scattering / Segmental Mixing /
\end{abstract}

Tapered block polymers of styrene and isoprene were prepared by a simultaneous copolymerization of the two monomers containing trace amounts of tetrahydrofuran, with $s$-BuLi as the initiator and benzene as the polymerization solvent. The sequence distribution of the two monomers and the supermolecular structure were investigated in our previous paper. ${ }^{1}$ The results obtained may be summarized as follows. Pyrolysis gaschromatography and proton NMR spectroscopy indicated that the two monomers in the tapered block polymer chains are considerably mixed with each other and give, at least from a phenomenological view point, a block polymer consisting of a "styrene-rich block sequence" and an "isoprene-rich block sequence."

Owing to the mixing of the two monomers in the primary structure, the repulsive interaction between the two block sequences (i.e., styrene-rich and isoprene-rich sequences) is much weaker than that between the pure polystyrene and polyisoprene block chains in ideal block polymers (i.e., conventional block polymers prepared by sequential polymerization). This effect leads to an enhanced mixing of the two block sequences in phase-

a Present Address: Department of Synthetic Chemistry, Faculty of Engineering, Nagoya University, Nagoya 464, Japan.

b Present Address: Hyogo University of Education, Hyogo 673-14, Japan.

* To whom correspondence should be addressed. 
Table I. Characterization of tapered and ideal block polymers and homopolymers

\begin{tabular}{|c|c|c|c|c|c|c|}
\hline $\begin{array}{l}\text { Specimen } \\
\text { code }\end{array}$ & $\begin{array}{c}M_{n} \times 10^{-3} \\
\quad(\text { total) }\end{array}$ & $M_{w} / M_{n}$ & $\begin{array}{l}\mathrm{wt} \% \text { of } \\
\text { styrene }\end{array}$ & $\frac{M J^{\mathrm{a}}}{\%}$ & $\frac{Y(S)_{\mathrm{rel}}^{\mathrm{b}}}{\%}$ & Remarks \\
\hline TB-1 & 43 & 1.05 & 47 & 4.0 & 88.6 & Tapered block \\
\hline TB-2 & 63 & 1.08 & 48 & 4.0 & 88.3 & Tapered block \\
\hline TB-3 & 106 & 1.09 & 45 & 3.7 & 89.2 & Tapered block \\
\hline TB-5 & 97 & 1.18 & 51 & 0.24 & 84.9 & Ideal block* \\
\hline TB-6 & 49 & 1.13 & 45 & 0.17 & 84.9 & Ideal block \\
\hline HPS & 277 & 1.20 & 100 & - & - & Homopolymer \\
\hline HPI & 490 & 1.33 & 0 & - & - & Homopolymer \\
\hline
\end{tabular}

a Estimated minimum junctions.

b Relative styrene monomer yield calculated from eq 1 .

* Polymerized by a procedure slightly different from that for TB-6 (see ref 1).

separated microdomains. Thus, the microdomain structure of a tapered block polymer consists of a "styrene-rich phase" and an "isoprene-rich phase" as was clarified by our previous small-angle X-ray scattering and electron microscopic studies. ${ }^{1}$ This structure greatly differs from the microdomain structure of ideal block polymers of styrene and isoprene in which each phase consists almost solely of its own block sequence, i.e., the polystyrene (or polyisoprene) phase contains styrene (or isoprene) segments only.

In this paper, we describe the dynamic mechanical behavior of tapered block polymers in the linear region and its relation to the hetrophase structure of the polymers: microdomain structure, mixing of the two block sequences in each phase, and that in the domain-boundary interphase.

\section{TEST SPECIMENS}

Tapered block polymers TB-1 to TB-3 and ideal block polymers TB- 5 and 6 of styrene and isoprene as well as their homopolymers (HPS, HPI) were prepared by the methods described in a previous paper. ${ }^{1}$ Table I summarizes the results of characterization of these polymers. The parameters as well as the microstructure of polyisoprene were determined by the methods also described in the previous paper. ${ }^{1}$

The tapered block polymers contained, at least, 3.7 to $4.0 \%$ of the hybrid junctions of styrene and isoprene, (SI), along the molecular chain when estimated from pyrolysis gaschromatographic data.
The value for TB- 6 was very close to the theoretical junction of $0.18 \%$ calculated from the molecular weight of the ideal block. The values for TB-1 to TB-3 suggested that these polymers should have more than 4 junctions in 100 monomer units, so that the average sequence length is less than 25 monomer units.

The relative styrene monomer yield, $Y(\mathrm{~S})_{\mathrm{rel}}$, is defined by

$$
Y(\mathrm{~S})_{\mathrm{rel}}=Y(\mathrm{~S}) \times 100 /[Y(\mathrm{~S})+Y(\mathrm{SS})+Y(\mathrm{SSS})]
$$

since polystyrene degrades mostly into monomers, dimers, and trimers. Here, $Y(\mathrm{~S}), Y(\mathrm{SS})$, and $Y(\mathrm{SSS})$ are the yields of styrene monomer, dimer, trimer, respectively. The quantity $Y(\mathrm{~S})_{\mathrm{rel}}$ is an index of random monomer arrangement in the presence of the "boundary monomer effect" on degradation. ${ }^{1}$ Data of $Y(\mathrm{~S})_{\text {rel }}$ suggested that the styrene monomer units in TB-1 to TB-3 have more frequent contact with the counter monomer (isoprene) than those in TB-6.

Film specimens of the block polymers were prepared by casting about $5 \mathrm{wt} \%$ toluene solutions onto glass plates. The specimens were further dried under vacuum for a few weeks until their weight became constant, and were heat-treated at $100^{\circ} \mathrm{C}$ for 3 to $5 \mathrm{~h}$ prior to dynamic mechanical measurements.

\section{RESULTS AND DISCUSSION}

\section{Microdomain Structure}

Figure 1 shows the microdomain structure of the 


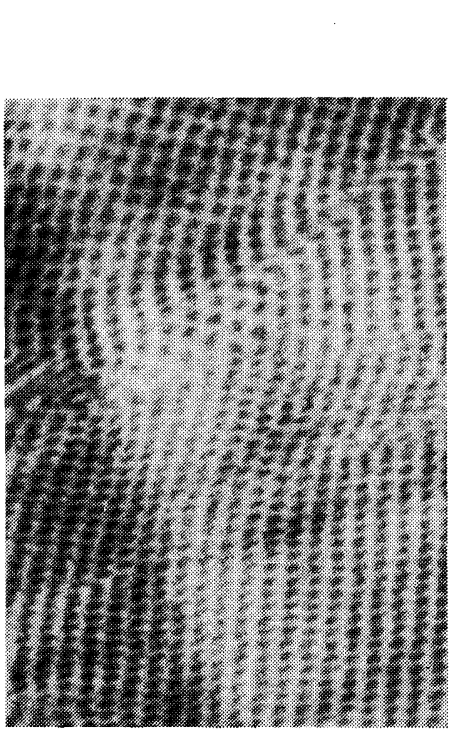

TB-1

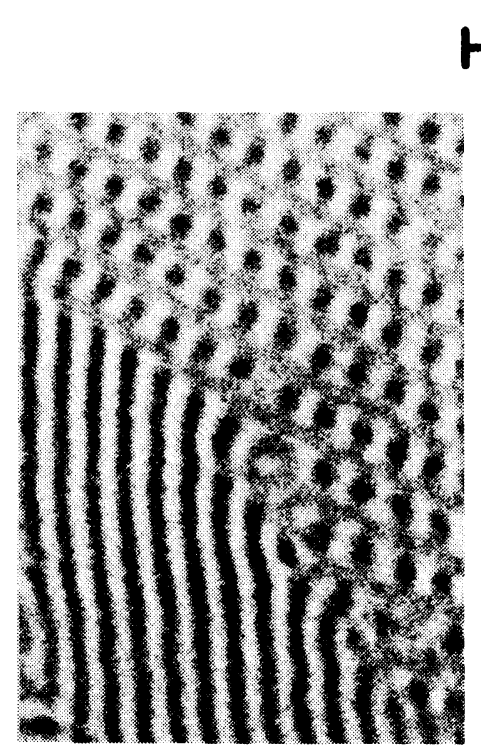

TB-2

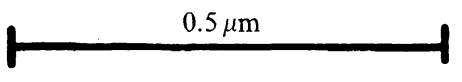



TB-3



TB-5

TB-6

Figure 1. Microdomain structure of the block polymers observed by transmission electron microscopy on ultrathin sections stained by $\mathrm{O}_{\mathrm{s}} \mathrm{O}_{4}$.

block polymers; these pictures were obtained by transmission electron microscopic observation of ultrathin sections stained by $\mathrm{OsO}_{4}$. Since the details are described elsewhere, ${ }^{1}$ we summarize the conclusions only. (1) The tapered block polymers TB-1 to 3 have microdomain structures as clear as the ideal block polymers TB-5 and 6. (2) However, each domain structure of the tapered polymers contains a substantial amount of dissimilar units, in contrast to that of the ideal block polymers. This enhanced mixing of the unlike units in the domains designated as styrene-rich and isoprene-rich domains was ap- 
parent from an extremely weak image contrast between the two domains under the microscope. (3) TB-1 and 2 have isoprene-rich rodlike domains dispersed in the styrene-rich matrix, while TB-3 to 6 have alternating lamellar microdomains. The finding that TB- 1 and 2 exhibited rod-like morphology in spite of their isoprene weight fractions being 0.53 and 0.52 , respectively, suggests that the mixing is
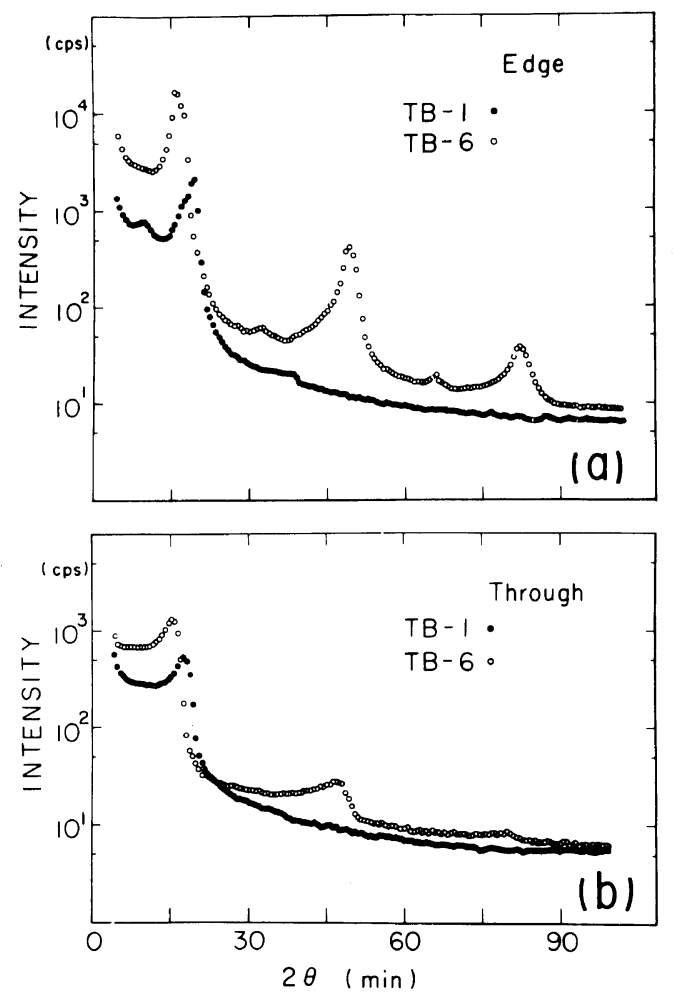

Figure 2. Typical SAXS profiles of the tapered block polymer (TB-1) and the ideal block polymer (TB-6). (a) "edge"-radiation and (b) "through"-radiation. $2 \theta$ is the scattering angle in min. arc $(\mathrm{Cu} K \alpha$-radiation). asymmetric. This means that the amount of isoprene monomers in styrene-rich blocks is larger than that of styrene monomers in isoprene-rich blocks. Such mixing leads to isoprene-rich block sequences shorter than styrene-rich block sequences.

The enhanced mixing was more quantitatively confirmed by small-angle X-ray scattering (SAXS) in the present paper. Figure 2 shows SAXS profiles of the tapered block TB- 1 and ideal block polymer TB-6, both having approximately the same molecular weight and chemical composition. Since the microdomains are preferentially oriented with respect to the normal to film surface, the SAXS profiles were taken with an incident beam normal ("through" radiation) and parallel to the film surface ("edge" radiation).

Figure 2 clearly shows that the tapered block polymer has a scattering power much weaker than the ideal block polymer. The weak scattering power is due to an decrease in mean-squared electron density fluctuation $\left\langle\eta_{\text {obs }}^{2}\right\rangle$ caused by the mixing mechanism described above. If $\rho_{\text {obs }}(\boldsymbol{r})$ and $\rho_{i}(\boldsymbol{r})$ represent the electron density profiles of the pseudo two-phase structure (with a sigmoidal density profile across the interface) and the ideal two-phase structure (with stepwise density profile across the interface), respectively (see Figure 3), we have

$$
\rho_{\text {obs }}(\boldsymbol{r})=\rho_{i}(\boldsymbol{r})^{*} h(\boldsymbol{r})
$$

where $h(\boldsymbol{r})$ is a function characterizing the density profile at the interphase and the asterisk designates the "convolution" operation. It follows that

$$
\begin{aligned}
\left\langle\eta^{2}\right\rangle_{\mathrm{obs}} & \equiv \stackrel{\frac{2}{\Delta \rho_{\mathrm{obs}}}}{(\mathbf{0})} \\
& =\left\langle\eta_{i}{ }^{2}\right\rangle \int \gamma_{i}(\boldsymbol{u}) \stackrel{2}{\tilde{h}}(-\boldsymbol{u}) d \boldsymbol{u}
\end{aligned}
$$

with

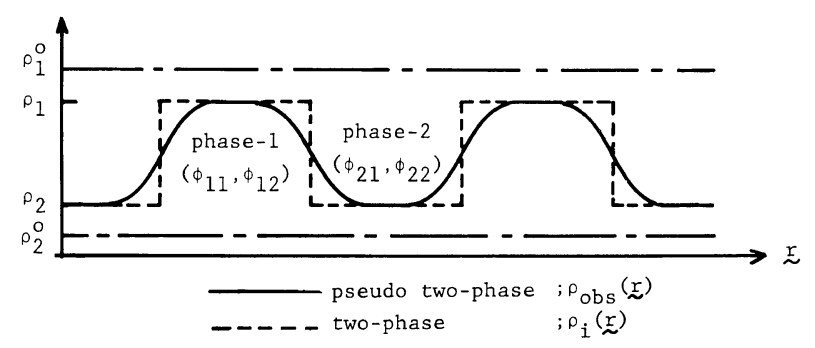

Figure 3. Schematic diagrams showing the electron density profiles $\rho_{\text {obs }}(\boldsymbol{r})$ and $\rho_{i}(\boldsymbol{r})$ for pseudo two-phase and two-phase structures, respectively. $\phi_{i j}$ is the volume fraction of $j$-monomeric units in the $i$-domain. 
Tapered Block Polymers

$$
\begin{aligned}
\left\langle\eta_{i}{ }^{2}\right\rangle & =\frac{2}{\Delta \rho}(\mathbf{0}) \\
\gamma_{i}(\boldsymbol{r}) & \equiv \frac{2}{\Delta \rho_{i}}(\boldsymbol{r}) / \frac{2}{\Delta \rho_{i}}(\mathbf{0})
\end{aligned}
$$

where the symbol $\stackrel{2}{\tilde{h}}$ designates the self-convolution of the function $h$, i.e.,

$$
\stackrel{2}{\tilde{h}}(\boldsymbol{r})=\int h(\boldsymbol{x}) h(\boldsymbol{r}+\boldsymbol{x}) \mathrm{d} \boldsymbol{x}
$$

and $\Delta \rho_{\text {obs }}(\boldsymbol{r})$ and $\Delta \rho_{i}(\boldsymbol{r})$ are the fluctuations in the electron density of the respective systems from average values. The mean squared electron density fluctuation $\left\langle\eta_{i}{ }^{2}\right\rangle$ for the two-phase structure depends on the extent of mixing, since

$$
\left\langle\eta_{i}^{2}\right\rangle=\left(\rho_{1}-\rho_{2}\right)^{2} \psi_{1} \psi_{2}
$$

where $\psi_{i}$ is the volume fraction of the microphase $i$ and $\rho_{i}$ is the electron density of the phase $i$ which depends on the extent of mixing. If $\phi_{i j}$ is the volume fraction of $j$-monomers in the $i$-phase and $\rho_{\mathrm{j}}{ }^{0}$ is the electron density of homopolymer $j$ (see Figure 3), we have

$$
\rho_{i}=\sum_{j=1}^{2} \phi_{i j} \rho_{j}^{0} \quad(i=1,2)
$$

with

$$
\sum_{j=1}^{2} \phi_{i j}=1 \quad(i=1,2)
$$

Thus, for a more extensive mixing (i.e., for greater $\left.\phi_{i j}, i \neq j\right)$, we obtain smaller values of $\left(\rho_{1}-\rho_{2}\right)^{2}$ and $\left\langle\eta_{i}{ }^{2}\right\rangle$, and hence a weaker scattering power $\left\langle\eta_{\text {obs }}^{2}\right\rangle$ (see eq 2), since

$$
\left(\rho_{1}-\rho_{2}\right)^{2}=\left(\phi_{11}-\phi_{21}\right)^{2}\left(\rho_{1}{ }^{0}-\rho_{2}{ }^{0}\right)^{2}
$$

Figure 4 shows the SAXS profiles of the tapered block polymers TB-1 to 3 (all edge-radiation). All these tapered block polymers have about the same scattering power, much lower than the ideal block polymers TB-5 and 6. The higher-order scattering maxima seen for the ideal block polymers in Figure 2 are less clear in the profiles of the tapered block polymers. This is due to the weak scattering power of these polymers and the enhanced background scattering arising from electron density fluctuations in polystyrene-rich and polyisoprene-rich phases, both attributable to the mixing effect. For TB-1 and 2 , broad, weak and overapping peaks are seen at the scattering angles equal to $\sqrt{3}$ and $\sqrt{4}$ times the angle for the first-order scattering maximum. These

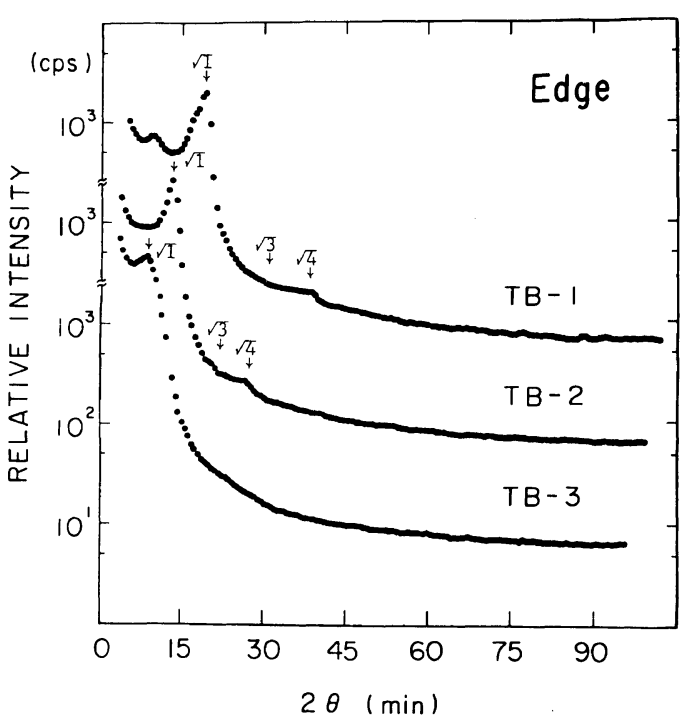

Figure 4. SAXS profiles of the tapered block polymers TB-1 to 3. "Edge"-radiation.

maxima probably arise from interparticle interference between the rodlike domains packed in the hexagonal lattice.

From the peak position $2 \theta_{\mathrm{m}}$ of the first-order scattering maximum, the Bragg spacing $D_{\mathbf{B}}$ can be calculated using the relation

$$
2 D_{\mathbf{B}} \sin \theta_{\mathrm{m}}=\lambda
$$

The Bragg spacing is equal to the identity period of alternating lamellar microdomains (domain spacing) or may be related to the nearest neighbor distance $D_{0}$ between the road packed in the hexagonal lattice by

$$
D_{0}=\sqrt{4 / 3} D_{\mathrm{B}}
$$

Figure 5 shows the domain spacing (either the lamellar identity period or the inter-rod distance) as a function of the total molecular weight for the tapered block polymers TB-1 to 3 and the ideal block polymers TB-5 and 6. The spacing for a series of lamellar microdomains (L-1 to L-6 in the previous paper $^{2}$ ) is also included in the figure. The graph clearly indicates that the molecular weight dependence of the domain spacing $D$ in the tapered block polymers follows the same line as that in the ideal block polymers. Moreover, it can be seen that the domain spacing of the rodlike microdomain is approximately equal to that of the lamellar micro- 
domains. The straight line shown has a slope of $2 / 3$, giving

$$
D \sim M_{n}^{2 / 3}
$$

which agrees with the finding from previous studies. $^{2-5}$

\section{Temperature for the Order-to-Disorder Transition}

Extensive mixing of unlike monomeric units in the primary structure should reduce the effective repulsive interaction between the styrene-rich and isoprene-rich block chains. If $f_{\mathrm{ss}}$ and $f_{\mathrm{SI}}$ are the mole fractions of styrene and isoprene monomers in the styrene-rich block chains, respectively, and $f_{\text {IS }}$ and $f_{\text {II }}$ the corresponding fractions in isoprene-rich block chains, and if the two monomers are randomly mixed in each block chain, the effective FloryHuggins parameter ${ }^{6} \chi_{\text {eff }}$ for the interaction between these two block chains may be given by ${ }^{7}$

$$
\begin{aligned}
\chi_{\text {eff }} & =\chi_{\text {sI }}\left(f_{\text {ss }}-f_{\text {SI }}\right)^{2} \\
& =\chi_{\text {sI }}\left(f_{\text {IS }}-f_{\text {III }}\right)^{2}
\end{aligned}
$$

where $\chi_{\mathrm{SI}}$ is the parameter for the interaction between polystyrene and polyisoprene.

For ideal block polymers, since $f_{\mathrm{SS}}=f_{\mathrm{II}}=1$ and $f_{\mathrm{SI}}=f_{\mathrm{IS}}=0, \chi_{\text {eff }}$ agrees with $\chi_{\mathrm{sI}}$. Thus $\chi_{\text {eff }}$ decreases with increasing degree of monomer intermixing. This decrease in $\chi_{\text {eff }}$ will lower the temperature $T_{\mathrm{c}}$ of the order-to-disorder transition, i.e., the temperature at which the microdomains become a homogeneous mixture. This transition takes place when $\chi_{\text {eff }}$ reaches a critical value $\left(\chi_{\text {eff }}\right)_{c}$ given by

$$
\left(\chi_{\text {eff }}\right)_{\mathrm{c}}=\left[\frac{\left(\delta_{\mathrm{S}}-\delta_{\mathrm{I}}\right)^{2}}{\rho_{0} k_{\mathrm{B}} T}\right]_{\mathrm{c}}\left(f_{\mathrm{SS}}-f_{\mathrm{SI}}\right)^{2} \sim \frac{\left(f_{\mathrm{SS}}-f_{\mathrm{SI}}\right)^{2}}{T_{\mathrm{c}}}
$$

Therefore, $T_{\mathrm{c}}$ decreases with increasing monomer intermixing.

Figure 6 shows the variation in the SAXS profiles of TB-1 with temperature. With increasing temperature, the scattering maximum due to regular domain spacing becomes lower and its position shifts toward larger angles. Upon cooling from 200 to $28^{\circ} \mathrm{C}$, the original profile at room temperature was recovered. Changes in scattered intensity and domain spacing with temperature are illustrated in Figure 7 for TB-1, TB-2, and TB-3. For TB-1, the reciprocal scattered intensity at a particular scattering angle near $2 \theta_{\mathrm{m}}$ decreases linearly with increasing reciprocal absolute temperature above $150^{\circ} \mathrm{C}$. The Bragg



Figure 5. Domain spacings (the lameller identity period or the inter-rod distance) as a function of total molecular weight for the tapered block polymers TB- 1 to 3 and the ideal block polymers TB-5 and 6. The spacings for a series of the lamellar microdomains (designated as L-1 to 6 in the previous paper ${ }^{2}$ ) are also included. L-3 and L-5 correspond to TB-6 and TB-5, respectively.

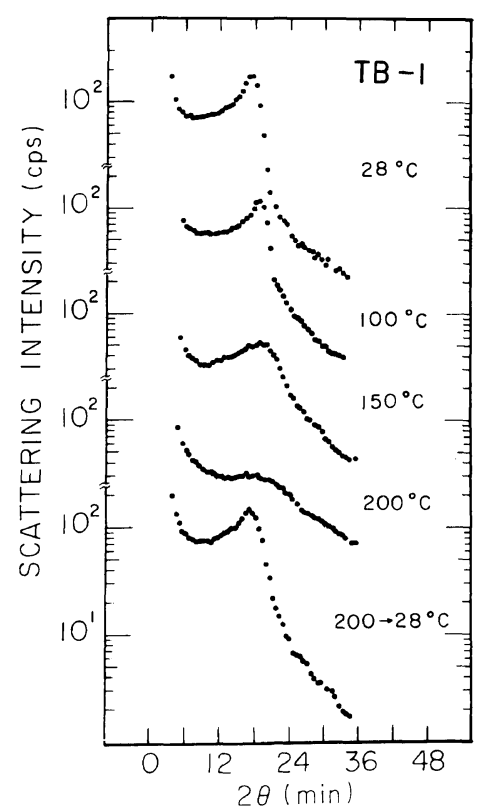

Figure 6. Variation in SAXS profiles for TB-1 with temperature.

spacing is nearly independent of temperature in the range where this linear relation holds. With decreasing temperature below $150^{\circ} \mathrm{C}$, the spacing tends to increase, and deviations from the linear relation become large. These changes are considered to 


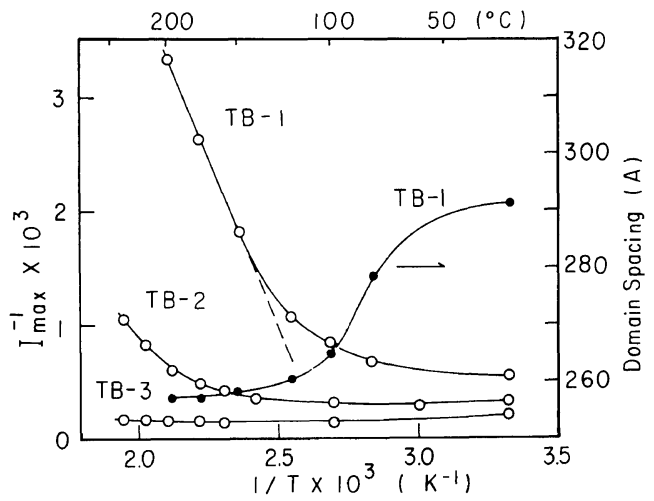

Figure 7. Variation in the domain spacing and the scattered intensity at a given scattering angle $2 \theta \simeq 2 \theta_{\max }$ with reciprocal absolute temperature for TB-1 to 3 .

indicate the onset of microphase separation, i.e., the disorder-to-order transition. The change in SAXS profile accompanying the thermal transition was first discussed by Roe et al. ${ }^{8}$ and extensively so in our previous papers. ${ }^{4,5,9}$ The scattering from the disordered phase was analyzed on the basis of Leibler's theory. ${ }^{10}$

According to Leibler, ${ }^{10}$ the scattering intensity $I(q)$ of a bulk block polymer in the disordered state is given by

$$
I(q) \sim Z[F(x)-2 \chi Z]^{-1}
$$

where $q=(4 \pi / \lambda) \sin \theta, 2 \theta$ and $\lambda$ being the scattering angle and wavelength of the incident beam, respectively. The function $F(x)$ is defined in eq IV-6 of ref 10 . If $\chi$ is given by

$$
\chi=A+B / T
$$

eq 14 yields

$$
I^{-1}(q) \sim\left[\frac{F(x)}{N}-2 A\right]-\frac{2 B}{T}
$$

Therefore, $I^{-1}$ should linearly decrease with $T^{-1}$ in the disordered state. Deviations from linearity at lower $T$ may be attributed to the onset of microphase separation. Thus, $T_{\mathrm{c}}$ for TB-1 is estimated to be about $150^{\circ} \mathrm{C}$. Theoretically, the intensity $I$ increases with lowering temperature (according to eq 14) and diverges to infinity at the spinodal temperature $T_{\mathrm{s}}$ (i.e., $I^{-1}$ approaches zero at $T_{\mathrm{s}}$ ). However, our experimental results indicate, as shown for TB-1, that the decrease in $I^{-1}$ with $T^{-1}$ becomes progressively slower and $I^{-1}$ never reaches zero. The increase in domain spacing with a decrease in temperature in the low temperature region is the result of increasing segregation of the constituting block chains, as discussed in detail in our previous papers. ${ }^{4,5,11}$

For TB-2 and 3, no linear relationship between $I^{-1}$ and $T^{-1}$ was observed in the temperature range treated in this experiment (up to $240^{\circ} \mathrm{C}$ ). Hence, the transition temperatures for these polymers were probably higher than $240^{\circ} \mathrm{C}$, and should become higher as the molecular weight of the block polymer increases. The temperature $T_{\mathrm{c}}$ for TB-6, which has a molecular weight and chemical composition approximately equal to that of TB-1, was higher than $220^{\circ} \mathrm{C}$ as shown in our previous paper. ${ }^{1}$ Thus, the reduction in the effective interaction parameter $\chi_{\text {eff }}$ due to intermixing of unlike units should lower $T_{\mathrm{c}}$ very effectively. It should be noted that rheological behavior in the flow region was found to be extremely sensitive to the transition temperature $T_{\mathrm{c}}$; the flow was typically Newtonian above $T_{\mathrm{c}}$ and non-Newtonian below $T_{\mathrm{c}}{ }^{27-35}$ Although the rheological behavior in the flow region is beyond the scope of this paper, it is relevant to point out that flow behavior is greatly affected by intermixing of unlike units in the primary structure of tapered block polymers.

\section{Dynamic Mechanical Response}

Figure 8 shows the real $\left(E^{\prime}\right)$ and imaginary parts $\left(E^{\prime \prime}\right)$ of dynamic tensile moduli at $10 \mathrm{~Hz}$ as a function of temperature for a series of block polymers. The ideal block polymers TB- 5 and 6 show two primary dispersions associated with the microbrownian motion of polyisoprene and polystyrene phases (typical of the two-phase structure). On the other hand, the tapered block polymers (TB-1 to 3) exhibit a single broad primary dispersion maximum at a temperature between those of the corresonding homopolymers, despite the fact that these polymers have the microphase-separated domain structures shown in Figure 1. Although the responses of TB-1 to 3 are quite similar in the temperature range shown in Figure 8, they should exhibit important differences at higher temperature, since their transition temperatures $T_{\mathrm{c}}$ are different as mentioned in the previous section.

A close look at the $E^{\prime \prime}$ profiles of the tapered block polymers indicates that each broad dispersion 

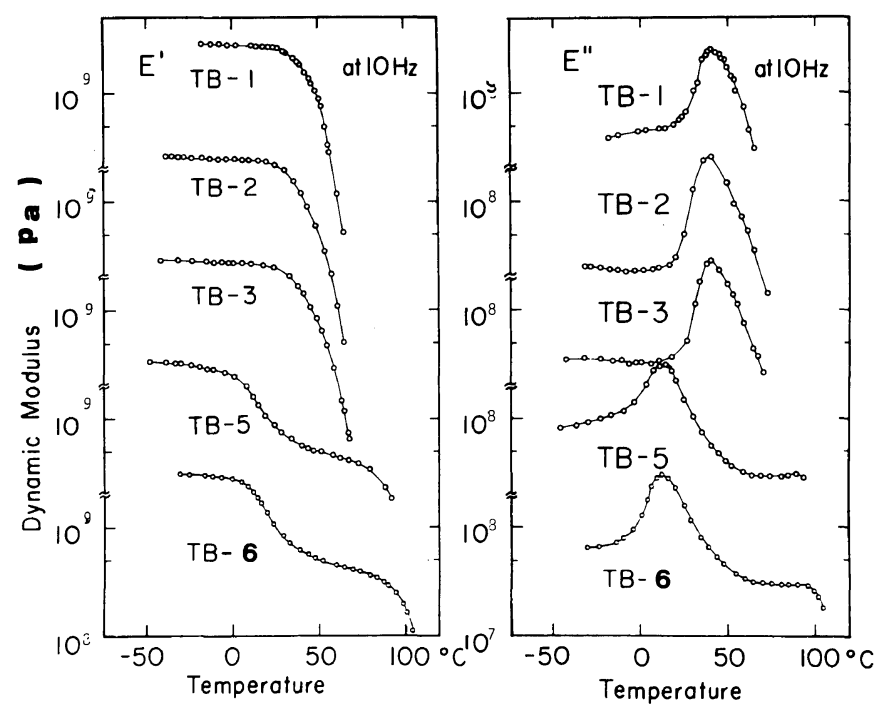

Figure 8. Real and imaginary parts of dynamic tensile moduli at $10 \mathrm{~Hz}$ as a function of temperature for a series of block polymers.

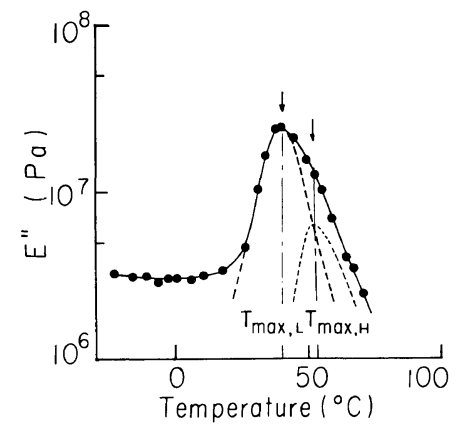

Figure 9. Resolution of the dispersion maximum of $E^{\prime \prime}$ into those corresponding to "isoprene-rich" and "styrene-rich" microdomains for TB-3, and the definitions of $T_{\max , \mathrm{L}}$ and $T_{\max , \mathrm{H}}$.

maximum consists of two primary dispersions as illustrated in Figure 9: one, corresponding to isoprene-rich microdomains, has a maximum $E^{\prime \prime}$ at $T_{\max , \mathrm{L}}$ and the other, corresponding to styrene-rich microdomains, has a maximum $E^{\prime \prime}$ at $T_{\max , \mathrm{H}}$. The estimated values of $T_{\max , \mathrm{L}}$ and $T_{\max , \mathrm{H}}$ are listed in the last column of Table II. If $T_{\max , \mathrm{L}}$ and $T_{\max , \mathrm{H}}$ obey Fox's equation ${ }^{12}$ for the glass transition temperature of a binary polymer mixture, the composition $\phi_{i j}$ of the $j$-component in the $i$-phase may be estimated from

$$
T_{\max , \mathrm{L}}^{-1}=\frac{\phi_{\mathrm{I}, \mathrm{I}}}{T_{\max , \mathrm{L}}^{0}}+\frac{\phi_{\mathrm{I}, \mathrm{S}}}{T_{\max , \mathrm{H}}^{0}}
$$

$$
T_{\max , \mathrm{H}}^{-1}=\frac{\phi_{\mathrm{S}, \mathrm{I}}}{T_{\max , \mathrm{L}}^{0}}+\frac{\phi_{\mathrm{S}, \mathrm{S}}}{T_{\max , \mathrm{H}}^{0}}
$$

where

$$
\begin{aligned}
& \phi_{\mathrm{I}, \mathrm{I}}+\phi_{\mathrm{I}, \mathrm{S}}=1 \\
& \phi_{\mathrm{S}, \mathrm{I}}+\phi_{\mathrm{S}, \mathrm{S}}=1
\end{aligned}
$$

and $T_{\max , \mathrm{L}}^{0}$ and $T_{\max , \mathrm{H}}^{0}$ are, respectively, the temperatures at which $E^{\prime \prime \prime}$ s for homopolyisoprene (HPI) and homopolystyrene (HPS) become maxima at $10 \mathrm{~Hz}\left(T_{\max , \mathrm{L}}^{0}=283^{\circ} \mathrm{K}\right.$ and $\left.T_{\max , \mathrm{H}}^{0}=378^{\circ} \mathrm{K}\right), \phi_{\mathrm{I}, \mathrm{I}}$ and $\phi_{\mathrm{I}, \mathrm{S}}$ are the weight fractions of isoprene monomers and styrene monomers in isoprene-rich domains, respectively, and $\phi_{\mathrm{S}, \mathrm{I}}$ and $\phi_{\mathrm{S}, \mathrm{S}}$ are the corresponding quantities in styrene-rich domains.

It should be noted that eq 17 and 18 assume a random mixing of the components in each phase. The values of $\phi_{i j}$ thus estimated are also presented in Table II, indicating clearly that each domain in the ideal block polymers is essentially pure and contains few unlike monomeric units. On the other hand, each domain in the tapered block polymers comprises a significant amount of unlike units as seen from the values of $\phi_{\mathrm{IS}}$ and $\phi_{\mathrm{SI}}$. The mixing of unlike units is asymmetric $\left(\phi_{\mathrm{IS}}<\phi_{\mathrm{SI}}\right)$, which means that the amount of isoprene in styrene-rich domains is larger than that of styrene in isoprene-rich domains. A similar asymmetric intermixing was found for tapered block polymers consisting of styrene 
Tapered Block Polymers

Table II. Domain structure of tapered and ideal block polymers

\begin{tabular}{|c|c|c|c|c|c|c|c|c|c|}
\hline \multirow{4}{*}{$\begin{array}{l}\text { Sample } \\
\text { code }\end{array}$} & \multirow{4}{*}{ Morphology } & \multirow{2}{*}{\multicolumn{2}{|c|}{$\frac{\text { Domain spacing }}{\mathrm{nm}}$}} & \multirow{2}{*}{\multicolumn{4}{|c|}{$\frac{\text { Composition of each domain }}{\mathrm{b}}$}} & \multirow{2}{*}{\multicolumn{2}{|c|}{$\frac{T_{\max } \text { of } E^{\prime \prime} \text { at } 10 \mathrm{~Hz}}{{ }^{\circ} \mathrm{C}}$}} \\
\hline & & & & & & & & & \\
\hline & & \multirow[b]{2}{*}{$D_{\mathrm{EM}}$} & \multirow[b]{2}{*}{$D_{\mathrm{SAXs}}{ }^{\mathrm{a}}$} & \multicolumn{2}{|c|}{ Isop-rich domain } & \multicolumn{2}{|c|}{ Sty-rich domain } & \multirow{2}{*}{$\begin{array}{l}\text { Isop-rich } \\
\text { domain } \\
\left(T_{\max , \mathrm{L}}\right)\end{array}$} & \multirow{2}{*}{$\begin{array}{l}\text { Sty-rich } \\
\text { domain } \\
\left(T_{\max , \mathrm{H}}\right)\end{array}$} \\
\hline & & & & $\begin{array}{c}\text { Isop } \\
\phi_{11}\end{array}$ & $\begin{array}{l}\text { Sty } \\
\phi_{\text {IS }}\end{array}$ & $\begin{array}{c}\text { Isop } \\
\phi_{\mathrm{SI}}\end{array}$ & $\begin{array}{l}\text { Sty } \\
\phi_{\text {ss }}\end{array}$ & & \\
\hline TB-1 & Rod & 29 & $33.3(28.8)$ & 0.62 & 0.38 & $0: 50$ & 0.50 & 40 & 51 \\
\hline TB-2 & Rod & 37 & $40.5(35.1)$ & 0.63 & 0.37 & 0.46 & 0.54 & 39 & 54 \\
\hline TB-3 & Lamellar & 53 & 52.9 & 0.62 & 0.38 & 0.47 & 0.53 & 40 & 53 \\
\hline TB-5 & Lamellar & 46 & 46.3 & 0.97 & 0.03 & 0.11 & 0.89 & 12 & 91 \\
\hline TB-6 & Lamellar & 29 & 31.9 & 0.97 & 0.03 & 0.08 & 0.92 & 12 & 95 \\
\hline
\end{tabular}

a Estimated from SAXS edge view patterns.

b Estimated irom $T_{\max }$ for $E^{\prime \prime}$ using the Fox equation.

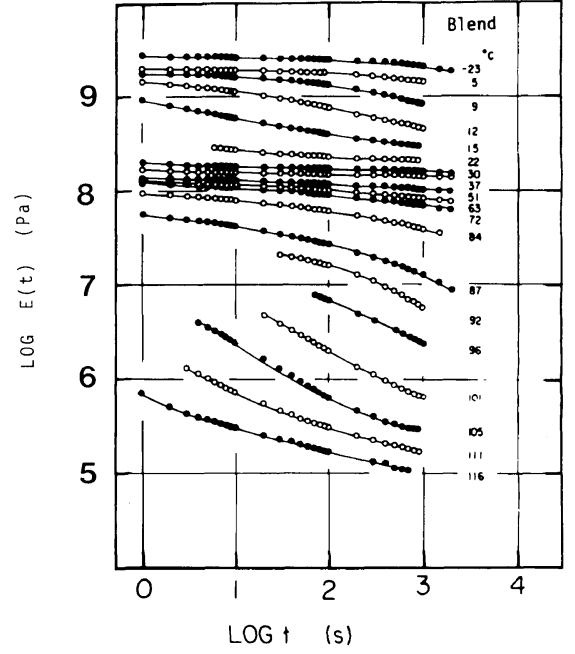

(a)

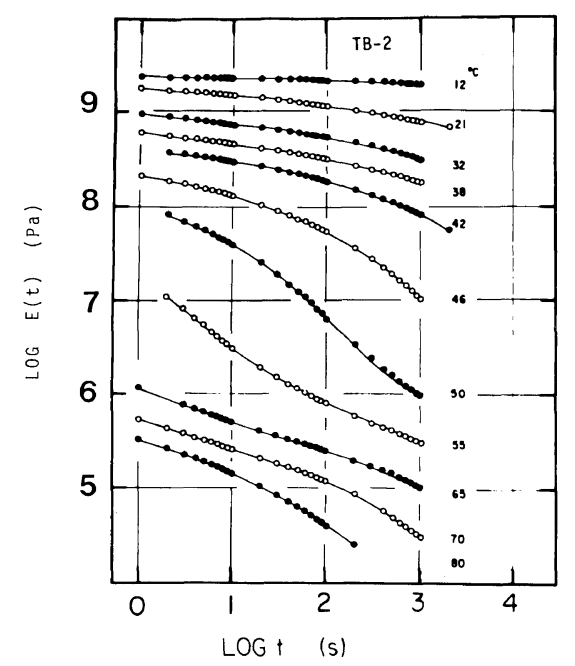

(b)

Figure 10. Typical stress relaxation curves at various temperatures for (a) the polymer blend (1:1 mixture of HPS and HPI) and for (b) the tapered block polymer TB-2.

and butadiene (the $\mathrm{P}$-series in our previous paper ${ }^{13}$ ). The conclusion obtained here is consistent with that from electron microscopy (Figure 1) and SAXS (Figure 4).

Figure 10 shows typical tensile stress relaxation curves at various temperatures. The physical blend of HPS and HPI (a 1:1 mixture by weight) and the ideal block polymer as well, though the data for the latter are not shown, clearly show two stress relaxation processes at about 10 and $100^{\circ} \mathrm{C}$. These processes can be attributed to the micro-brownian motion of HPI and HPS, which is typical of systems having a two-phase structure. On the other hand, the tapered block polymers exhibit a single relaxation process spread around $50^{\circ} \mathrm{C}$, as illustrated for TB-2.

Isothermal stress relaxation curves can be superimposed only by a horizontal shift. Typical "apparent" master curves obtained by this operation are shown in Figure 11, and the shift factor $a_{\mathrm{T}}$ in Figure 12, where $T_{\mathrm{g}, \mathrm{L}}$ is the glass transition temperature of the polyisoprene domain or the isoprene-rich domain.

Figure 11 shows "apparent" master stress re- 


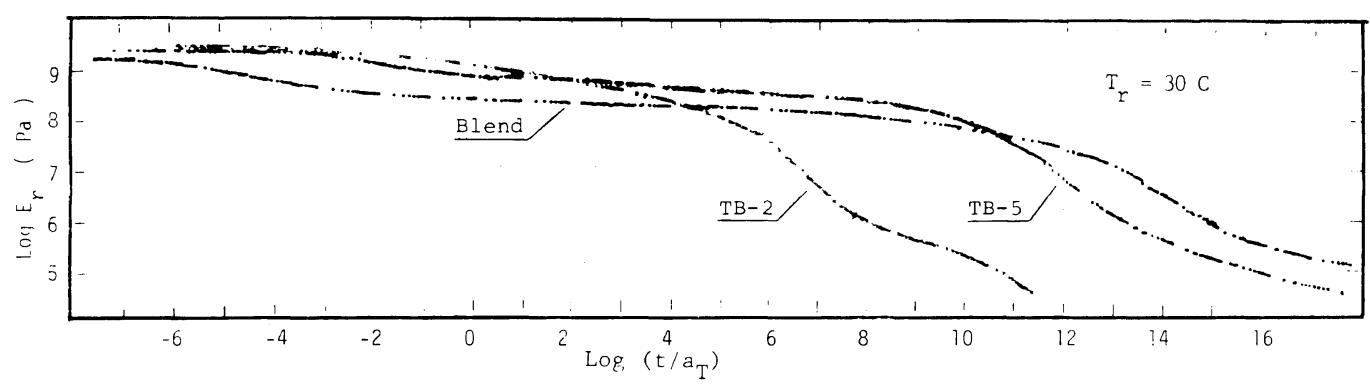

Figure 11. Typical "apparent" master stress relaxation curves for the polymer blend, ideal block polymer TB-5, and tapered block polymer TB-2 at a reduce temperature $T_{\mathrm{r}}=30^{\circ} \mathrm{C}$.



Figure 12. Shift factor $a_{\mathrm{T}}$ as a function of temperature $T-T_{\mathrm{g}, \mathrm{L}}$ where $T_{\mathrm{g}, \mathrm{L}}$ is the glass transition temperature of the polyisoprene domain or "isoprene-rich" domain for the tapered block polymers TB-1 and 2, ideal block polymer TB-5, polymer blend and HPI.

laxation curves for a physical blend (Blend), a tapered block (TB-2), and an ideal block (TB-5) reduced to $30^{\circ} \mathrm{C}$. The physical blend shows two distinct relaxation processes; one at about $10^{-4} \mathrm{~s}$ and the other at about $10^{15} \mathrm{~s}$, each associated with the microbrownian motion of HPI and HPS in respective phases. The ideal block polymer TB-5 also shows two distinct relaxation processes at about $10^{-2}-10^{-1}$ and $10^{13} \mathrm{~s}$. The difference of the two relaxation times of TB-5 from those of the polymer blend and also the fact that the relaxation modulus of TB- 5 decays, in the time scale between the two primary relaxations, more rapidly than that of the physical blend may be attributed to the "domain-boundary relaxation" that has been discussed in detail elsewhere. ${ }^{13,14}$ This is the relaxation due to dynamic interactions of the chains in the respective domains at the domain-boundary interphase. ${ }^{15-20}$ The tapered block polymer TB-2 shows a broad relaxation centered at about $10^{4}$ $10^{5} \mathrm{~s}$. This relaxation may be a superposition of the two relaxation processes at about $10^{\circ}-10^{1}$ and $10^{6}-10^{7} \mathrm{~s}$, which are associated, respectively, with the isoprene-rich and styrene-rich domains.

The temperature dependence of the shift factor $a_{\mathrm{T}}$, especially that for the primary relaxation of the polyisoprene or isoprene-rich domain, was analyzed using the WLF equation. ${ }^{21}$ The results are summarized in Table III, where the characteristic parameters $C_{1}{ }^{\mathrm{g}}, C_{2}{ }^{\mathrm{g}}, f_{\mathrm{g}} / B$, and $\alpha_{\mathrm{f}} / B$ have the same meaning as explained in Ferry's book. ${ }^{21} T_{\mathrm{d}}$ is the temperature at which the experimental $\log a_{\mathrm{T}}$ for the polyisoprene primary relaxation begins to deviate from the WLF equation (see Figure 12):

$$
\log a_{\mathrm{T}}=-C_{1}{ }^{\mathrm{g}}\left(T-T_{\mathrm{g}, \mathrm{L}}\right) /\left(C_{2}{ }^{\mathrm{g}}+T-T_{\mathrm{g}, \mathrm{L}}\right)
$$

It should be noted that $T_{\mathrm{d}}$ for the ideal block polymers is lower than that for the physical blend. This suggests that some relaxations other than the primary relaxation of polyisoprene are activated at lower temperature for the ideal block than for the physical blend. Probably, such relaxations are associated with the domain-boundary relaxation. Since the block polymer has an interfacial area and interfacial volume much larger than the polymer blend, the domain-boundary relaxation in block polymers should occur more intensively than in physical blends.

It is interesting to note that the tapered block polymers respond as if they were structurally homogeneous, in the sense that the temperature dependence of their shift factors obeys the WLF equation throughout the entire temperature range, 
Table III. Parameters characterizing temperature dependence of $a_{\mathrm{T}}$ for tapered block polymers, ideal block polymer and polymer blend

\begin{tabular}{|c|c|c|c|c|c|c|}
\hline Polymer & $\frac{T_{\mathrm{g}}}{{ }^{\circ} \mathrm{C}}$ & $\frac{T_{\mathrm{d}}}{{ }^{\circ} \mathrm{C}}$ & $C_{1}{ }^{\mathrm{g}}$ & $\frac{C_{2}{ }^{\mathrm{g}}}{\operatorname{deg}}$ & $f_{\mathrm{g}} / B$ & $\frac{\alpha_{\mathrm{f}} / B}{\mathrm{deg}^{-1}}$ \\
\hline TB-1 & 40 & - & 13.4 & 27.7 & 0.032 & $1.2 \times 10^{-3}$ \\
\hline TB-2 & 39 & - & 14.2 & 31.5 & 0.031 & $9.7 \times 10^{-4}$ \\
\hline TB-5 & 15 & 70 & 18.6 & 40.0 & 0.023 & $5.8 \times 10^{-4}$ \\
\hline Blend & 10 & 92 & 20.4 & 41.8 & 0.021 & $5.1 \times 10^{-4}$ \\
\hline HPI & 0 & - & 12.2 & 22.0 & 0.036 & $1.6 \times 10^{-3}$ \\
\hline
\end{tabular}

although they have the microdomain structures as discussed in the previous section. Further, their characteristic parameters $C_{1} \mathrm{~g}, C_{2} \mathrm{~g}, f_{\mathrm{g}} / B$, and $\alpha_{\mathrm{g}} / B$ are close to those of homopolyisoprene, although $T_{\mathrm{g}, \mathrm{L}}$ 's are higher than that of HPI owing to extensive segmental intermixing.

\section{Features of Structural Models}

In Figure 13, structural models for (a) the physical blend, (b) the ideal block polymers (TB-5 and 6), and (c) the tapered block polymers (TB-1 to 3 ) are depicted on the basis of the results from electron microscopic, SAXS, and dynamic mechanical studies. Here are shown the spatial distributions of relative segment densities $\tilde{\rho}_{\mathrm{A}}$ and $\tilde{\rho}_{\mathrm{B}}$ for $\mathrm{A}$ and $\mathrm{B}$ monomeric units in an $\mathrm{AB}$ polymer blend, an $\mathrm{AB}$ diblock polymer, and an $\mathrm{AB}$ tapered block polymers. The relative segmental density $\left(\tilde{\rho}_{\mathrm{A}}\right)$ for $\mathrm{A}$ is defined as $\tilde{\rho}_{\mathbf{A}}=\rho_{\mathbf{A}}(z) / \rho_{\mathbf{A}}{ }^{0}$, where $\rho_{\mathbf{A}}(z)$ and $\rho_{\mathbf{A}}{ }^{0}$ are the number densities of A-units in the system and in a pure bulk polymer A, respectively. Since any polymeric liquid is essentially incompressible, $\tilde{\rho}_{\mathrm{A}}(z)+\tilde{\rho}_{\mathrm{B}}(z)=1$ in the equilibrium state.

When $\mathrm{A}$ and $\mathrm{B}$ are highly segregative as in pairs of styrene and isoprene, the A and B phases in the physical blend and also in ideal block polymers consist essentially of pure A and B respectively. The unlike units are extensively mixed only in the interfacial region of thickness $\lambda_{1}$ or $\lambda_{2}$, where $\lambda_{1}$ is somewhat smaller than $\lambda_{2}{ }^{22}$ Although $\lambda_{1}$ and $\lambda_{2}$ are not much different, the interfacial area and volume of the block polymer are far larger than those of the physical blend, ${ }^{23}$ leading to a mechanical response of the block polymers which is more sensitively influenced by the domain-boundary relaxation than that of the physical blend. ${ }^{24}$

In contrast to the domain in the block polymers,

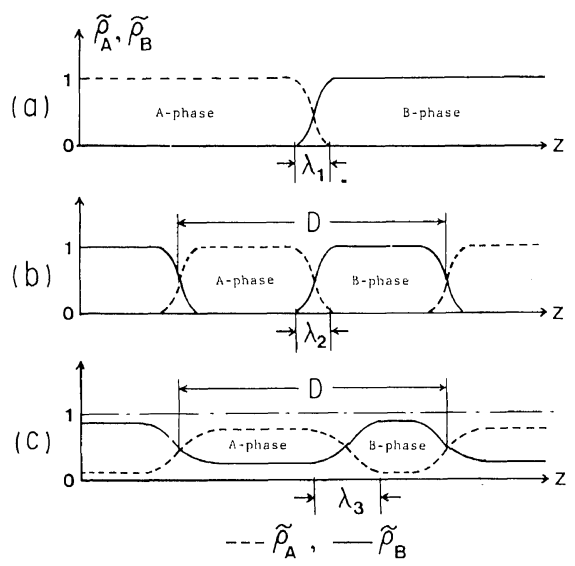

Figure 13. Structural models for (a) the polymer blend, (b) the ideal block polymers (TB-5 and 6), and (c) the tapered block polymers (TB-1 to 3 ) proposed on the basis of electron microscopic, SAXS, and dynamic mechanical data.

that in the tapered block polymers contains a significant amount of unlike segments as seen from Table II. Hence, there is no pure phase in them which satisfies $\tilde{\rho}_{\mathbf{K}}=0$ or $1(K=\mathrm{A}$ or $\mathrm{B})$. The mixing of unlike segments in the respective domains may not always be symmetric and varies with the mode of segmental mixing in the primary structure. Figure 13(c) shows a case where the degree of mixing of $B$ units in the A domain is larger than that of $\mathrm{A}$ units in the $\mathrm{B}$ domain. It was also found that the domain spacings were approximately equal in the tapered block polymers and the ideal block polymers when the molecular weights were the same.

Enhanced mixing of unlike segments in the primary structure gives rise to a thicker interphase 


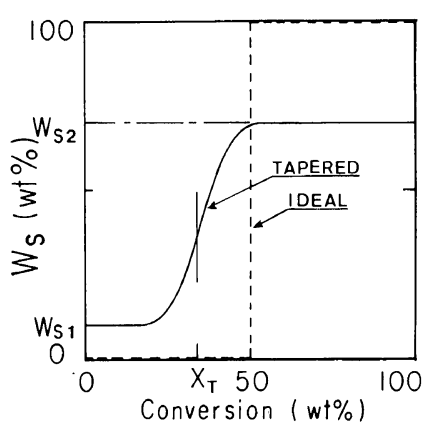

Figure 14. Schematic diagram showing variation in the instantaneous weight percent of styrene units $W_{\mathrm{s}}$ as a function of conversion $X$ of the tapered block polymers (solid line) and ideal block polymers (broken line) having $1: 1$ composition by weight.

$\lambda_{3}\left(>\lambda_{2}\right)$, since it lowers the parameter $\chi_{\text {eff }}$ for the effective interaction between the A-rich and B-rich block chains. Thus, segmental intermixing has a two-fold effect on the mechanical response. (i) The response is affected by the segmental intermixing in the domains (defined as "mixing-in-domain" effect) and (ii) by that in the domain-boundary interphase (defined as "mixing-in-domain boundary" effect or "domain-boundary mixing" effect). We analyzed these two effects on dynamic mechanical responses in our previous paper, ${ }^{13}$ and found that, for the particular tapered block polymers studied here, the "mixing-in-domain" effect outweighs the "domainboundary mixing" effect. Owing to the enhanced mixing, the tapered block polymers respond mechanically as if they were structurally homogeneous and thus are characterized by the WLF equation, though they have a microheterogeneous structure.

Figure 14 shows the segmental intermixing in the primary structure predicted from electron microscopic, SAXS, and dynamic mechanical studies. Here, the instantaneous composition of styrene $W_{\mathrm{S}}$ is plotted against the weight fractional conversion $\mathrm{X}$ of the polymer. For the ideal block polymer having $50 \mathrm{wt} \%$ polystyrene, $W_{\mathrm{S}}$ may be 0 (or 100 ) for $0 \leq X<50$ and 100 (or 0 ) for $50<X \leq 100$. For the tapered block polymers having $50 \mathrm{wt} \%$ styrene, however, $W_{\mathrm{S}}$ changes in a stepwise fashion: $W_{\mathrm{S} 1}$ for for $0<X<X_{\mathrm{T}}$ and $W_{\mathrm{S} 2}$ for $X_{\mathrm{T}}<X<100$. Thus, the tapered block polymer may be approximated, as a first approximation, by a block polymer consisting of an isoprene-rich random copolymer having a composition $\left(W_{\mathrm{S} 1}, 1-W_{\mathrm{S} 1}\right)$ and a styrene-rich random copolymer having a composition $\left(W_{\mathrm{S} 2}\right.$, $1-W_{\mathrm{S} 2}$ ). If segmental intermixing takes place more extensively in the styrene-rich copolymer than in the isoprene-rich copolymer, the block length of the isoprene-rich copolymer should be shorter than that of styrene-rich copolymer $\left(X_{\mathrm{T}}<50\right)$. This speculation was verified for a particular tapered block polymer of styrene and butadiene by Kraus et al. ${ }^{25}$ and Hashimoto et al. ${ }^{13}$

In our previous paper, we carried out computer simulation of mixing-in-domain and domainboundary mixing effects on the dynamic mechanical response using the composite model of Takayanagi. ${ }^{26}$ The approach was basically the same as employed by Kraus and Rollmann. ${ }^{19}$ The results indicated that segmental mixing in the domainboundary alone is not sufficient to produce the observed broad temperature dispersion maximum of $E^{\prime \prime}$. Enhanced mixing of unlike segments was necessary to account for such a dispersion maximum. The present work showed that the parameters $\phi_{\mathrm{II}}=0.6$ and $\phi_{\mathrm{SS}}=0.5$ and the volume fraction 0.25 of the interphase give a satisfactory fit to the isochronal data for the tapered block polymers TB-1 to 3 .

Acknowledgements. This work was partially suppoted by Grants-in-Aid for Scientific Research under Grant No. 56490013 (addressed to T. H.) and for Special Project Research under Grant No. 57119003 ("Design of Multiphase Biomedical Materials") from the Ministry of Education, Science and Culture of Japan.

\section{REFERENCES}

1. Y. Tsukahara, N. Nakamura, T. Hashimoto, H. Kawai, T. Nagaya, Y. Sugimura, and S. Tsuge, Polym. J., 12, 455 (1980). (Part I of this series.)

2. T. Hashimoto, M. Shibayama, and H. Kawai, Macromolecules, 13, 1237 (1980).

3. T. Hashimoto, M. Fujimura, and H. Kawai, Macromolecules, 13, 1660 (1980).

4. T. Hashimoto, M. Shibayama, and H. Kawai, Macromolecules, 16, 1093 (1983); Polym. Prepr., Am. Chem. Soc., Div. Polym. Chem., 23(1), 21 (1982).

5. T. Hashimoto, Proceedings of IUPAC 28th Symposium on Macromolecules, University of Massachusetts, Amherst, MA., 1982, p 705; Macromolecules, 15, 1548 (1982).

6. P. J. Flory, "Principles of Polymer Chemistry," 
Cornell University Press, Ithaca, New York, N. Y., 1967.

7. R. J. Roe and W. C. Zin, Macromolecules, 13, 1221 (1980).

8. R. J. Roe, M. Fishkis, and C. J. Chang, Macromolecules, 14, 1091 (1981).

9. M. Shibayama, T. Hashimoto, and H. Kawai, Macromolecules, 16, 16 (1983).

10. L. Leibler, Macromolecules, 13, 1602 (1980).

11. M. Shibayama, T. Hashimoto, H. Hasegawa, and H. Kawai, Macromolecules, 16, 1427 (1983).

12. T. G Fox, Bull. Am. Phys. Soc., 1(3), 123 (1956).

13. T. Hashimoto, Y. Tsukahara, K. Tachi, and $\mathrm{H}$. Kawai, Macromolecules, 16, 648 (1983). (Part IV of this series.)

14. Y. Tsukahara, A. Izumi, T. Hashimoto, and $\mathrm{H}$. Kawai, Polym. J., 14, 887 (1982).

15. S. L. Aggarwal, R. A. Livigni, L. F. Marker, and T. J. Dudek, "Block and Graft Copolymers," J. J. Burke and V. Weiss, Ed., Syracuse University Press, New York, N. Y., 1973, pp 157-194.

16. M. Shen and D. H. Kaelble, J. Polym. Sci., B, 8, 149 (1970).

17. D. G. Fesko and N. W. Tschoegl, Intern. J. Polym. Mater., 3, 51 (1974).

18. T. Soen, M. Shimomura, T. Uchida, and H. Kawai, Colloid \& Polym. Sci., 252, 933 (1974).

19. G. Kraus and K. W. Rollmann, J. Polym. Sci., Polym. Phys. Ed., 14, 1133 (1976).

20. J. Bares, Macromolecules, 8, 244 (1975).
21. J. D. Ferry, "Viscoelastic Properties of Polymers," Wiley, New York, N. Y., 1980, Chapter 11.

22. E. Helfand, Macromolecules, 8, 552 (1975).

23. H. Hashimoto, M. Fujimura, T. Hashimoto, and $\mathrm{H}$. Kawai, Macromolecules, 14, 844 (1981).

24. A. Todo, H. Uno, K. Miyoshi, T. Hashimoto, and H. Kawai, Polym. Eng. Sci., 17, 587 (1977).

25. G. Kraus and K. W. Rollmann, Angew. Makromol. Chem., 16/17, 271 (1971).

26. M. Takayanagi, H. Harima, and Y. Iwata, J. Soc. Mater. Sci. Jpn., 12, 389 (1963).

27. C. I. Chung and J. C. Gale, J. Polym. Sci., Polym. Phys. Ed., 14, 1149 (1976).

28. E. V. Gouinlock and R. S. Porter, Polym. Eng. Sci., 17, 534 (1977).

29. E. R. Pico and M. C. Williams, Polym. Eng. Sci., 17, 573 (1977).

30. C. I. Chung and M. I. Lin, J. Polym. Sci., Polym. Phys. Ed., 16, 545 (1978).

31. C. I. Chung, H. L. Griesbach, and L. Young, $J$. Polym. Sci., Polym. Phys. Ed., 18, 1237 (1980).

32. J. M. Widmaire and G. C. Meyer, J. Polym. Sci., Polym. Phys. Ed., 18, 2217 (1980).

33. G. Kraus and T. Hashimoto, J. Appl. Polym. Sci., 27, 1745 (1982).

34. H. Watanabe, T. Kotaka, T. Hashimoto, M. Shibayama, and H. Kawai, J. Rheol., 26, 153 (1982).

35. T. Hashimoto, M. Shibayama, H. Kawai, H. Watanabe, and T. Kotaka, Macromolecules, 16, 361 (1983). 